\title{
A Practical Approach for Pathophysiological Diagnosis of Refractory Hypokalemia
}

\author{
Mohamed Foda Hendi, Zeyad Faoor Alrais, Hesham El Kholy
}

Department of Intensive Care Medicine, Rashid Hospital, Dubai health Authority, Dubai, United Arab Emirates

\begin{abstract}
Hypokalemia is a common finding in hospitalized patients. In most cases the cause will be obvious. However, in a subgroup of patients the cause is occasionally uncertain and establishing the diagnosis may present difficulties and can become a challenge.

In such cases, measurement of urinary indices e.g. trans-tubular potassium gradient [TTKG] and random urine potassium/creatinine concentrations along with blood acid/base parameters and assessment of urinary excretion of potassium, chloride, calcium and creatinine have been employed in the differential diagnosis.

TTKG and urine potassium/creatinine ratio are used as a rapid appropriate index of potassium excretion and to establish a proper diagnosis and management in this medical emergency. It helps to differentiate between hypokalemia caused by redistribution or renal potassium wasting.

Urinary chloride excretion and urinary calcium/creatinine ratio in conjunction with plasma acid/base values, aldosterone, renin and cortisol values are used for narrowing the differential diagnosis of refractory hypokalemia.

Conclusions: Calculating the trans-tubular potassium concentration gradient, urinary potassium/creatinine ratio, urinary chloride excretion and urinary calcium/creatinine ratio in conjunction with plasma acid/base values provided simple and reliable tests for differential diagnosis of causes of refractory hypokalemia and to provide a proper management in the intensive care unit and in the emergency until advanced further tests to be done.
\end{abstract}

\section{Keywords:}

Refractory Hypokalaemia, Trans-tubular Potassium Concentration Gradient, Urinary Potassium-creatinine Ratio, Urinary Calcium-Creatinine Ratio, Bartter Syndrome, Gitelman Syndrome.
*Correspondence to Author:

Mohamed Foda Hendi

Department of Intensive Care Medicine, Rashid Hospital, Dubai health Authority, Dubai, United Arab Emirates

How to cite this article:

Mohamed Foda Hendi, Zeyad Faoor Alrais, Hesham El Kholy.A Practical Approach for Pathophysiological Diagnosis of Refractory Hypokalemia. Global Journal of Urology and Nephrology, 2018, 1:6

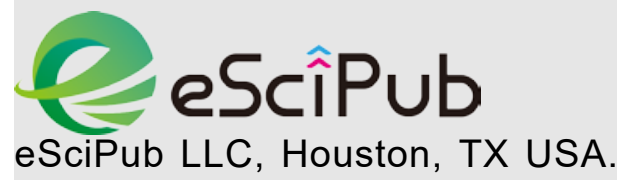

Website: http://escipub.com/ 


\section{Discussion:}

Hypokalemia is a common clinical problem which the etiological diagnosis can frequently be based on the patient history and the clinical situation. (1) The cause can usually be determined from the history such as hypokalemia due to vomiting, diarrhoea, or diuretic use and no further investigation will be necessary. However, in a subgroup of patients the cause is occasionally uncertain and establishing the diagnosis may present difficulties. (2)

In situations where the cause of hypokalemia is not clear, measurement of urinary potassium excretion and assessment of blood pressure and acid-base values are often helpful. (3)

The causes of hypokalemia can be simply divided into two categories: the one condition includes diseases causing renal potassium wasting such as diabetic ketoacidosis, Cushing's syndrome, primary hyperaldosteronism, Bartter syndrome, Gitelman syndrome, hypomagnesemia, and renal tubule acidosis, as well as intake of loop and thiazide diuretics, which results in $\mathrm{K}$ loss by the kidneys; and the other consists of those leading to extra-renal excretion or transient shifting of $\mathrm{K}$ into cells, as in thyrotoxic periodic paralysis, exogenous insulin infusion, hyperinsulinemia, vomiting, and diarrhoea. (4)

The traditional approach to differentiate between renal and extrarenal causes of hypokalemia is based on urinary potassium excretion measured in 24 hours urine samples or random urinary potassium concentration values. A urinary potassium concentration that is more than 15 to $20 \mathrm{mmol} /$ day or more than 15 to $20 \mathrm{mmol} / \mathrm{L}$ suggests that there are renal causes for potassium wasting. Hypokalemia in which these values are less than $15 \mathrm{mmol} /$ day or $15 \mathrm{mmol} / \mathrm{L}$ respectively indicates that there are extrarenal causes for potassium depletion. Obtaining a 24 hours potassium excretion rate is not practical in a medical emergency because potassium replacement must be given promptly which can compromise the accuracy of 24 hours urinary potassium. ${ }^{(5)}$

A spot urine collection before potassium replacement should be used to help for establishing the diagnosis. ${ }^{(5)}$ In our patient there was high urinary potassium concentration (58.9 $\mathrm{mmol} / \mathrm{L}$ ) that indicates there are renal causes for potassium loss. However, random measurements may be misleading as the urine potassium concentration is determined by both the amount of potassium in the urine and the urine volume. (6) Usually there are many overlapping values of the spot urinary potassium concentration as polyuria is common in patients with hypokalemia. The polyuria may be due to thirst or defective renal concentration in patients with chronic hypokalemia which leads to a low value of the urinary potassium concentration even if a significant renal potassium loss is present. Therefore, a more reliable test is required. (5)

The trans-tubular potassium concentration gradient (TTKG) is an index reflecting the degree of potassium excretion in the cortical collecting ducts of the kidneys and indirectly assessing mineralocorticoid bioactivity in patients who have hypo- or hyperkalemia. (7) (8) Measurement of the TTKG is considered superior to measurement of urinary potassium alone for assessing the contribution of renal excretion to potassium levels. ${ }^{(9)}$ TTKG are useful in the pathophysiological differential diagnosis of hypokalemia. ${ }^{(1)}$

The trans-tubular potassium gradient estimates the ratio of potassium in the tubular fluid at the end of the cortical collecting tubule to that in the peritubular capillaries and can be estimated from:

TTKG $=$ (urine potassium $\times$ Osmolality plasma $) /$ (plasma potassium $\times$ Osmolality urine). For this formula to be accurate, urine osmolality must exceed plasma osmolality and urine sodium should be greater than $25 \mathrm{mmol} / \mathrm{L}$. (7) (8)

The normal renal response when hypokalemia is due to non-renal causes is a trans-tubular potassium gradient less than 2 which indicates GJUN:http://escipub.com/global-journal-of-urology-and-nephrology/ 
intracellular shift of potassium. while a transtubular potassium gradient more than 3 indicates hypokalemia of renal origin (renal potassium wasting). (9)

However, there are recent studies that advise to shift in emphasis from the TTKG to other variable reflecting potassium excretion e.g. the urinary potassium/creatinine ratio due to intrarenal urea recycling which leads to a higher rate of renal excretion of potassium. (10) (11)

The other variable reflecting potassium excretion, the urinary potassium/creatinine ratio $(\mathrm{U}(\mathrm{K} / \mathrm{Cr})$, has been used to evaluate the cause of hypokalemia. (5) Measurement of the urinary potassium/creatinine ratio is preferred because it is not influenced by the urine volume. $\mathrm{U}(\mathrm{K} / \mathrm{Cr})$ may be a valuable marker to distinguish between renal and extra-renal K loss. (4) (6)

Many studies have shown that the urinary potassium/creatinine ratio can be used to differentiate between hypokalemia caused by renal potassium loss and hypokalemia caused by extrarenal potassium depletion, as found in ion channelopathy hypokalemic periodic paralysis, by intracellular shift of serum potassium into skeletal muscle. (4) (6) (12)

The efficacy of the urinary potassium/creatinine ratio was evaluated in a previous study of fortythree patients with severe hypokalemia associated with paralysis. The urinary potassium/creatinine ratio was able to differentiate between the thirty patients with hypokalemic periodic paralysis (HPP) (whose hypokalemia was caused by an internal shift of extracellular potassium into the cells) and the thirteen patients with hypokalemia mostly due to renal potassium wasting (Ten of them had renal tubular acidosis or Gitelman syndrome). The urinary potassium/creatinine ratio was significantly lower in the patients with periodic paralysis. Hypokalemia and paralysis may be due to short term shift of potassium into cells in HPP or due to a large deficit of potassium in nonHPP. (5)
Failure to differentiate between hypokalemic periodic paralysis from non hypokalemic periodic paralysis may lead to improper management. It is critical to distinguish hypokalemia caused by redistribution, to avoid the risk that these patients may have rebound hyperkalemia after potassium replacement due to reversal of the shift of extracellular potassium into the cells. ${ }^{(5)}$

It is important to make the diagnosis promptly because different therapies are required for each type. The differential diagnosis can be challenging as the clinical features of HPP and non-HPP are almost indistinguishable. Many patients with non-HPP have been misdiagnosed as having HPP. (5)

The diagnostic cut off value for the potassium/creatinine ratio was approximately $1.5 \mathrm{mmol} / \mathrm{mmol}$ (some authors say 2.5 $\mathrm{mmol} / \mathrm{mmol}$ or $22 \mathrm{mmol} / \mathrm{g}$ ). In addition, patients with periodic paralysis had normal acid-base balance, while patients without periodic paralysis often had metabolic alkalosis or metabolic acidosis. (5)

Measurement of urinary indices such as such as excretory rate of $\mathrm{K}_{+}$(TTKG) and random urine $(\mathrm{K} / \mathrm{Cr})$ concentrations along with blood acidbase parameters have been employed in the pathophysiological diagnosis. (1)

A random urinary potassium/creatinine ratio $(\mathrm{K} / \mathrm{Cr})$ less than $1.5(\mathrm{mmol} / \mathrm{mmol})$ suggests a transcellular potassium shift into cells, poor potassium intake or gastrointestinal potassium loss. If hypokalemia is associated with acute paralysis with absence of acid base disorder, we should consider familial, sporadic or thyrotoxic periodic paralysis. While metabolic acidosis with urinary $\mathrm{K} / \mathrm{Cr}$ ratio less than 1.5 suggests lower gastrointestinal loss due to diarrhoea, enteric fistula or laxative abuse. However, some patients with diarrhoea due to laxative abuse develop metabolic alkalosis. ${ }^{(3)}$ (13) (14)

While metabolic acidosis with urinary $\mathrm{K} / \mathrm{Cr}$ ratio more than 1.5 is often due to diabetic 
ketoacidosis or distal renal tubular acidosis type 1 or type 2. (3)

There are many differential causes of hypokalemia due to renal loss with metabolic alkalosis and by measurement of urinary chloride level, we can able to differentiate the causes of hypokalemia due to renal loss (urinary $\mathrm{K} / \mathrm{Cr}$ ratio more than 1.5) with metabolic alkalosis. Patients with hypokalemia due to renal loss (high $\mathrm{K} / \mathrm{Cr}$ ratio) with metabolic alkalosis can be divided into two groups based on their urinary chloride excretion. The possible causes of low urine chloride level (< $10 \mathrm{mmol} / \mathrm{L})$ are vomiting, post hypercapnic alkalosis, nasogastric suction and congenital chloride diarrhoea. (13) (14)

Patients with high urine chloride level (> 20 $\mathrm{mmol} / \mathrm{L}$ ) can be separated into two groups based on their arterial blood pressure. Patients with high urinary chloride excretion (> $20 \mathrm{mmol} / \mathrm{L}$ ) and high arterial blood pressure include many differential diagnosis which can subdivided by measurement of serum aldosterone levels, renin activity and serum cortisol levels can help subdivide these patients. The groups with high serum renin and aldosterone levels include secondary aldosteronism, malignant hypertension, renal artery stenosis, renin secreting tumor and diuretic therapy in patients with essential hypertension. The causes of hypokalemia associated with metabolic alkalosis and hypertension with low renin and high aldosterone levels include primary hyperaldosteronism (Conn's syndrome or congenital adrenal hyperplasia), adrenal carcinoma. ${ }^{(13)}$

The presence of a non-aldosterone mineralocorticoid or mineralocorticoid like substance should be suspected in any patients with hypokalemia, hypertension and metabolic alkalosis with low serum aldosterone and renin activity levels. (13)

Measurement of serum cortisol levels can help to subdivide these patients. Those who have high serum cortisol levels are due to Cushing's syndrome, ACTH producing pituitary adenoma, ectopic ACTH syndrome (e.g, caused by small cell lung carcinoma) and exogenous steroids. Those who have normal serum cortisol levels are due to Liddle's syndrome, apparent mineralocorticoid excess syndrome, or drugs

with mineralocorticoid or glucocorticoid effects such as licorice ${ }^{(13)}$

While patients with high urinary chloride excretion (> $20 \mathrm{mmol} / \mathrm{L}$ ) and normal arterial blood pressure are caused by diuretics, Gitelman syndrome and Bartter syndrome. Diuretic therapy is the most common cause of hypokalemia which can be excluded by history. Diuretic induced hypokalemia is usually but not always associated with a mild to moderate metabolic alkalosis. (13)

The primary defect in both Gitelman syndrome and Bartter syndrome is an impairment in one of the transporters involved in sodium chloride reabsorption in the distal tubule and Henle's loop, respectively. The tubular defects in sodium chloride transport produce a clinical disorder that appears similar to that seen with chronic ingestion of a loop diuretic (mimicking Bartter syndrome) or a thiazide diuretic (mimicking Gitelman syndrome). (15)

\section{BARTTER SYNDROME VERSUS GITELMAN SYNDROME}

Bartter syndrome (BS) is a hereditary condition transmitted as an autosomal recessive (Bartter type 1 to 4 ) or dominant trait (Bartter type 5). There is an inherited defect in the renal tubules that causes low potassium levels, low chloride levels, which in turn causes metabolic alkalosis with varying degrees of hypercalciuria. (16) (17) It is a consequence of abnormal function of the kidney, which become unable to properly regulate the volume and composition of body fluids due to mutations in renal tubular transporters in the thick ascending limb of loop of Henle which lead to defective reabsorption of sodium and chloride. A first consequence of the tubular defect in Bartter Syndrome is polyuria. ${ }^{(16)}$ These patients appear similar to that seen with patients of chronic ingestion of a loop diuretic. 
Bartter Syndrome is characterized by hypokalemia of renal origin with renal potassium and salt wasting, metabolic alkalosis, hypercalciuria, normal arterial blood pressure, high plasma renin and aldosterone levels. ${ }^{(13)}$

Type 3 Bartter syndrome patients have the mildest presentation. (18) The classical form or type 3 Bartter syndrome is associated with hypomagnesemia in $20 \%$ of cases and normal or increased calciuria. This form is related to mutations of CLCNKB gene encoding for a chloride channel in Henle's loop. (15) (19) Clinically, Bartter Syndrome type 3 and 5 appear to be less severe than types 1 and 2, despite similar biochemical indices. (16)

Patients with Bartter syndrome types 1, 2 and 4 presents at a younger age than classic Bartter syndrome type 3. Patients with classic Bartter syndrome type 3 present later in life and may be sporadically asymptomatic or mild symptomatic. (16) (18)

Most patients of Bartter Syndrome are usually symptomatic early in life, they present with symptoms often severe in the neonatal period, although occasional patients, mostly Bartter Syndrome type 3 and rare patients with Bartter Syndrome type 4 and 5, may continue into adulthood with few if any manifestations. (16) (20)

In contrast, Gitelman Syndrome (GS) always presents in adult life. It is a much more common disease and less severe than Bartter Syndrome. (6) These patients appear similar to that seen with patients of chronic ingestion of thiazide diuretics. It is due to a defect in the gene encoding for the thiazide sensitive sodium/chloride cotransporter and it is associated with hypocalciuria and hypomagnesemia. (13) The degree of hypokalemia is usually mild to moderate in Gitelman Syndrome. Recently, however, more patients with Gitelman Syndrome have been found with a degree of hypokalemia that is similar to that in patients with Bartter syndrome. Many patients of Gitelman Syndrome in Asia present with paralysis related to severe hypokalemia. ${ }^{(5)}$
The diagnosis of Gitelman Syndrome is based on the following criteria: hypokalaemia due to renal potassium loss, hypomagnesemia of renal origin, and hypocalciuria with a urine calcium/creatinine ratio less than 0.1 $\mathrm{mmol} / \mathrm{mmol}(0.2 \mathrm{mg} / \mathrm{mg})$. (5) No evidence supports the need for 24 hours urine collection, spot urine samples are usually sufficient to establish the diagnosis. (21)

Urinary calcium excretion is important because it differentiate between the two syndromes. In contrast to the hypocalciuria of Gitelman syndrome, Bartter syndrome patients are often documented to have hypercalciuria. (18)

Although 24 hours collection of urine calcium is best, random urine calcium measurement in relation to creatinine can be performed. The normal reference for the urine calcium $(\mathrm{mg} / \mathrm{dl})$ : urine creatinine $(\mathrm{mg} / \mathrm{dl})$ ratio is less than 0.14 . Values exceeding 0.2 are found in patients with hypercalciuria. (22)

Hypercalciuria predisposes Bartter Syndrome patients for nephrolithiasis and nephrocalcinosis (calcification of the renal tissue). (16)

The persistent steady form of hypokalemia in BS and GS may suddenly become life threatening under certain aggravating conditions. Physicians need to be cognizant of such renal tubular disorders and promptly treat patients at risk. (18)

Distinguishing between BS and GS is not always straightforward due to phenotypic variance. The genetic diagnosis is now possible but there are several limitations including unavailability and cost. As mentioned before, GS is typically associated with hypocalciuria and BS with hypercalciuria, although some patients with type 3 Bartter syndrome have normal urinary calcium excretion. (18) (20) (23)

Conclusions: Calculating the trans-tubular potassium concentration gradient, urinary potassium/creatinine ratio, urinary chloride excretion and urinary calcium/creatinine ratio in conjunction with plasma acid/base values provided simple and reliable tests for differential diagnosis of causes of refractory hypokalemia 
and to provide a proper management in the intensive care unit and in emergency until advanced further tests to be done.

\section{Conflict of Interest}

The author(s) declared no potential conflicts of interest with respect to the research, authorship, and/or publication of this article.

\section{Acknowledgement}

I want to thank all my co-authors for hard work and help.

\section{References}

1. Joo KW, Chang SH, Lee JG, Na KY, Kim YS, et al. (2000) Transtubular potassium concentration gradient (TTKG) and urine ammonium in differential diagnosis of hypokalemia. J Nephrol 13: 120-125.

2. Reimann D, Gross P (1999) Chronic, diagnosisresistant hypokalaemia. Nephrol Dial Transplant 14: 2957-2961.

3. Assadi $F$ (2008) Diagnosis of hypokalemia: A problem-solving approach to clinical cases. Iran J Kidney Dis 2: 115-122.

4. Lin C, Piao X, Pan Q, Li J, Shan Z, et al. (2017) Spot urine potassium creatinine ratio is a good alternative marker for 24 hours urine potassium in differential diagnosis of hypokalemia. Med Sci Tech 58:137-144.

5. Lin S, Lin Y, Chen DT, Chu P, Hsu CW, et al. (2004) Laboratory tests to determine the cause of hypokalemia and paralysis. Arch Intern Med 164: 1561-1566.

6. Viera AJ, Wouk N (2015) Potassium disorders: Hypokalemia and hyperkalemia. Am Fam Physician 92: 487-495.

7. Choi MJ, Ziyadeh FN (2008) The utility of the transtubular potassium gradient in the evaluation of hyperkalemia. J Am Soc Nephrol 19:424-426.

8. Lin SH (2008) A practical and pathophysiologic approach to hypokalemia. Hong Kong J Nephrol 10: 14-26.

9. Ethier JH, Kamel KS, Magner PO, Lemann J, Halperin ML (1990) The transtubular potassium concentration in patients with hypokalemia and hyperkalemia. Am J Kidney Dis 15: 309-315.

10. Halperin ML (2017) Assessing the renal response in patients with potassium disorders: A shift in emphasis from the TTKG to the urine $\mathrm{K}+$ /creatinine ratio. Afr J Nephrol 20: 22-24.

11. Kamel KS, Halperin ML (2011) Intrarenal urea recycling leads to a higher rate of renal excretion of potassium: Hypothesis with clinical

implications. Curr Opin Nephrol Hypertens 20: 547-554.

12. Colussi G (2002) Bartter syndrome type 3: An unusual cause of nephrolithiasis. Nephrol Dial Transplant 17: 521-523.

13. Lim S (2007) Approach to hypokalemia acta med indones-indones. J Intern Med 39: 56-64.

14. Palmer B, Clegg DJ (2016) Physiology and pathophysiology of potassium homeostasis. Adv Physiol Educ 40: 480-490.

15. Konrad M, Weber S (2003) Recent advances in molecular genetics of hereditary magnesiumlosing disorders. J Am Soc Nephrol 14: 249-260.

16. Colussi G (2005) Bartter syndrome. Orphanet encyclopedia.

17. Soriano RJ (1998) Bartter and related syndromes: The puzzle is almost solved. Pediatr Nephrol 12: 315-327.

18. Fremont OT, Chan JCM (2012) Understanding Bartter syndrome and Gitelman syndrome. World J Pediatr 8: 25-30.

19. Vantyghem MC, Douillard C, Binaut R (1999) Bartter's syndromes. Ann Endocrinol 60: 465-472.

20. Zelikovic I, Szargel R, Hawash A, Labay V, Hatib I, et al. (2003) A novel mutation in the chloride channel gene, CLCNKB, as a cause of Gitelman and Bartter syndromes. Intern Soc Nephrol 63: 24-32.

21. Blanchard A, Bockenhauer D, Bolignano D, Calo LA, Cosyns E, et al. (2017) Gitelman syndrome: Consensus and guidance from a Kidney Disease: Improving Global Outcomes (KDIGO) controversies conference. Kidney Int 91: 24-33.

22. Foley KF, Boccuzzi L (2010) Urine calcium: Laboratory measurement and clinical utility. Lab Medicine 41: 683-686.

23. Cruz AJ, Castro A (2013) Gitelman or Bartter type 3 syndrome?: A case of distal convoluted tubulopathy caused by CLCNKB gene mutation. BMJ Case Rep 1: 1-4.

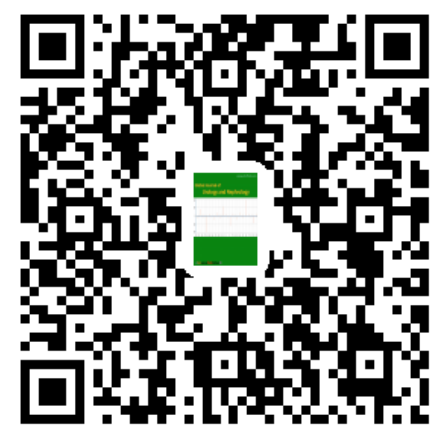

